\title{
Nonsmooth controllability theory and an example
}

\author{
T.D. Murphey, J.W. Burdick \\ Engineering and Applied Science, California Institute of Technology \\ Mail Code 104-44, Pasadena, CA 91125 USA \\ \{murphey, jwb\}@robotics.caltech.edu
}

\begin{abstract}
This paper extends recent results in local controllability analysis for Multiple Model Driftless Affine (MMDA) control systems. Such contrallability results can be interpreted as non-smooth extensions of Chow's theorem, and use a set-valued Lie Bracket. In particular, we formulate controllability in terms of generalized differential quotients (GDQs). Additionally, we present an extensive example in order to illustrate how these results can provide insight into the control of some specific physical systems. Moreover, this paper indicates that a multiple model system consisting of individually controllable models is not necessarily controllable.
\end{abstract}

\section{Introduction}

This paper considers the issue of controllability for systems of the following form:

Definition 1.1. A control system $\Sigma$ is said to be a multiple model driftless affine system (MIDA) if it can be expressed in the form

$\Sigma: \quad \dot{q}=f_{1}(q) u_{1}+f_{2}(q) u_{2}+\cdots+f_{n}(q) u_{n}$ where for any $q$ and $t, f_{i} \in\left\{g_{\alpha_{i}} \mid \alpha_{i} \in I_{i}\right\}$, with $I_{i}$ an index set, $f_{i}$ measurable in $(q, t), g_{\alpha_{i}}$ analytic in $(q, t)$ for all $\alpha_{i}$, and the controls $u_{i} \in \mathbb{R}$ are piecewise constant and bounded for all $i$.

Definition 1.1 implies the control vector fields may change, or switch, amongst a finite collection of vector fields, each representing a model, $P$, in a set of models $\mathcal{P}$. Such systems are intimately related to multiple model systems such as studied in $[6,13,14]$. However, we should emphasize that the "switching" is not like the switching phenomena found in $[2,10,4,20]$, or as typically studied in the hybrid control systems literature (e.g., $[15,1]$ ). In these studies, the switching is part of a control strategy to be implemented in the controller. Rather, it is switching induced by environmental factors, such as variations in the contact state between rigid bodies. Systems of this sort are actually quite common in engineering practice, and Section 3 develops an example in detail. As a first step in understanding such systems, we would like a local controllability test that works in the presence of a priori unknown switching behavior. This, as in the smooth case, is a first step towards eventually having generic tools for stabilization. This paper presents an application of a "discontinuous" version of the Chow's theorem that extends the classical result in a natural way to the systems of Def 1.1 .

This paper extends earlier work by the authors done in $[13,14]$. Prior to this work, there was little controllability analysis of such systems. As far as the authors are aware, two groups have studied controllability of nonsmooth systems like these. In [13] we used a setvalued lie bracket to study the local controllability of MMDA systems that experience arbitrarily fast switching in neighborhood of the operating point. In [14] we used a set-valued lie bracket to consider local controllability of MMIDA systems where the dynamics are determined by crossing a set of $C^{1}$ submanifolds of the configuration manifold. The current work extends the work done in [14] by taking away the need for a $C^{1}$ stratified system of manifolds forming the boundary of switching, and moreover uses a proof technique which has more potential in the future for developing stabilization techniques. While they did not study multi-model systems, [16] recently developed a nonsmooth version of Chow's theorem, using the same set-valued lie bracket, that applies to Lipschitz vector fields. The proofs found in this paper are adaptations of their proof methodology. These results are not appropriate for systems with switching, but nevertheless are important for some physical situations. An example of such a system is a carangiform fish swimming in a fluid with drag on the body (see [11]). The results obtained by [16] have strong analogues with our result, to the point that all three papers have essentially the same conditions for controllability, indicating that a set-valued lie bracket has wide potential application in the analysis of traditionally intractable systems. Moreover, the results presented in this paper represent the merging of their theory with our previous work.

In Section 2 we summarize these controllability results. Then, as an example of a physical system where these concepts are important, Section 3 analyzes a simple model of an overconstrained wheeled vehicle, which is inspired by novel high-mobility wheeled robots (e.g, 
the Mars Sojourner) that operate in rough terrain.

\section{Controllability Results}

We use several aspects of the formalism of [5] for investigating the properties of ODEs with discontinuous right hand sides. Eq. (1.1) can be viewed as a differential inclusion, i.e., a system of the form $\dot{q} \in \mathbf{f}$, where $\mathbf{f}$ is a set valued multi-function which is almost everywhere (a.e.) single valued. For equations of the form $\dot{q}=f(q)$ with $f$ discontinuous in $q$ at a point $q_{*}$, one must generally allow $f$ to take on the convex hull of limiting values $\lim _{q \rightarrow q_{*}} f$ at $q_{*}$ in order to guarantee existence of solutions (see [5, Chapter 2] for details). To account for this issue, we define the following at each $q$ :

$$
\mathbf{f}_{i}(q)=c o\left\{\lim _{q \rightarrow q_{*}} f_{i}(q)\right\}=c o\left\{g_{\alpha_{i}}\left(q_{*}\right) \mid \alpha_{i} \in I_{i}(q)\right\} \text {. }
$$

where $I_{i}(q)$ is an index on the set of limiting values of $f_{i}(q)$ at $q_{*}$, and $c o\{\cdot\}$ denote the convex hull of a set. For notational convenience, let $s_{i}\left(\mathbf{f}_{i}\right)$ denote a selection of $\mathrm{f}_{i}(q)$-i.e., a choice of a particular vector from $\mathbf{f}_{i}(q)$. Let $S_{i_{1}, i_{2}, \ldots, i_{k}}$ denote the set of all possible selections from $\mathbf{f}_{i_{1}}, \ldots, \mathbf{f}_{i_{k}}$.

Remark: We should comment more on the relationship between differential inclusions (DIs) and differential equations with discontinuous right hand sides (DEDRHSs). Consider the case $\dot{q}=f(q, t)$ with $f(q, t)=$ $\left\{f_{i}(q, t) \mid i \in I\right\}$ for some choice of $i$. When $f$ changes from $f_{i}$ to $f_{j}$, we typically must set $f=c o\left\{f_{i}, f_{j}\right\}$ at that point in order to guarantee existence of solutions. Moreover, $\dot{q}=f(q, t)$ implicitly depends on the choice of switching function determining $i$ at any given point call it $\sigma(q, t)$. An elementary result from $[5]$ is that the set of solutions to $\dot{q}=f(q, t)$ over all possible choices of $\sigma$ coincides with the set of solutions to $\dot{q} \in \mathbf{f}(q, t)$ where $\mathbf{f}=\operatorname{co}\left\{f_{i} \mid i \in I\right\}$. This, of course, is the relationship between DIs and DEDRHSs - for a given DEDRHS, we can choose a DI such that their solutions coincide.

Our result uses the notion of a set valued Lie bracket. This concept has its origin in two distinct areas. Previously in [13] we used a set-valued Lie bracket to consider the local controllability of MMDAs in the special case where switching occurs very rapidly. The use of a set-valued bracket was a natural consequence of the underlying assumptions in [13]. [16] uses a set-valued Lie bracket to prove the controllability of a driftless affine control system whose single-valued governing equation includes Lipschitz control vector fields. They showed that this choice of Lie bracket is a generalized differential quotient (GDQ) of the product of exponentials formulation of a lie bracket. Although these two applications seem different, the choice of Lie bracket is the same, and the resulting non-smooth versions of Chow's theorem are analogous. Hence, we use the following Lie bracket definition, adapted here to our situation:
Definition 2.1. Let $f_{1}$ and $f_{2}$ be as in Def 1.1. I.e., $f_{i} \in\left\{g_{\alpha_{i}} \mid \alpha_{i} \in I_{i}(q)\right\}$. The Lie bracket of $f_{1}$ and $f_{2}$ is defined as

$\left[f_{1}, f_{2}\right](q)=c o\left\{\lim _{j \rightarrow \infty}\left(D f_{1}\left(q_{j}\right) \cdot f_{2}\left(q_{j}\right)-D f_{2}\left(q_{j}\right) \cdot f_{1}\left(q_{j}\right)\right)\right\}$

for all sequences $\left\{q_{j}\right\}_{j \in \mathbb{N}}$ such that

1. $f_{1}$ and $f_{2}$ are differentiable $\forall q_{j}$,

2. $\lim _{j \rightarrow \infty} q_{j}=q$,

3. the limit exists.

Note that this lie bracket is a set valued object, which can be shown to be both compact and convex. It is moreover always well defined for MADA systems which are almost everywhere analytic. In the case where $\mathbf{f}=c o\left\{f_{i} \mid i \in I\right\}$ and $\mathbf{g}=c o\left\{g_{j} \mid j \in J\right\}$ (with $I$ and $J$ index sets), it is straightforward to show that $[\mathbf{f}, \mathbf{g}]=\operatorname{co}\left\{\left[f_{i}, g_{j}\right][i \in I, j \in J\}\right.$. Definition 2.1 is equivalent to a set-valued bracket defined previously in [12], where it was used to show local controllability for MMDA systems undergoing rapid switching. To analyze the controllability of MMDAs, we define:

Definition 2.2. Let $f_{i}$ be as in Def 1.1 and $\mathrm{f}_{i}$ as in Eq. (2.1). Define a distribution $\Delta_{s_{1} s_{2} \cdots s_{n}}(q)$ as

$\Delta_{s_{1} s_{2} \cdots s_{n}}(q)=\operatorname{span}\left\{v \mid v=s_{i}\left(\mathbf{f}_{i}(q)\right), \quad i=1 \ldots n\right\}$

That is, $\Delta_{s_{1} s_{2} \cdots s_{n}}(q)$ is formed from a particular selection of vectors from each $\mathbf{f}_{i}(q)$. Define the distribution $\Delta(q)$ as:

$$
\Delta(q)=\bigcap_{S_{1, \ldots, n}} \Delta_{s_{i} s_{2} \cdots s_{n}}(q)
$$

That is, $\Delta(q)$ is formed by intersecting the $\Delta_{s_{i} s_{2} \cdots s_{n}}(q)$ over all possible selections of $\mathbf{f}_{1}(q), \ldots, \mathbf{f}_{n}(q)$. Next define

$$
\Delta^{1}(q)=\bigcap_{S_{12,13, \ldots}}\left(\operatorname{span}\left\{v \mid v=s_{i j}\left(\left[\mathbf{f}_{i}, \mathbf{f}_{j}\right]\right)\right\}\right)
$$

(where $s_{i j}$ and $S_{12,13, \ldots}$ are defined similarly to before, with $s_{i j}$ being selections of $\left[\mathbf{f}_{i}, \mathbf{f}_{j}\right]$ and $S_{12,13, \ldots}$ being the set of all selections of $\left.\left[\mathbf{f}_{i}, \mathbf{f}_{j}\right]\right)$ and analogous higher order distributions formed from higher order set-valued lie brackets. Finally, define $\bar{\Delta}(q)$ as

$$
\bar{\Delta}(q)=\Delta(q) \bigcup \Delta^{1}(q) \bigcup \cdots .
$$

Now we cover some of the basic formalism found in [18] needed to prove our result. In particular, we need the notion of a $G D Q$ (defined below) which can be thought of as a generalized differential. We will use an open mapping theorem for $G D Q s$ from [17] to prove this extension of Chow's theorem. Let $\mathbb{N}$ denote the natural numbers. We denote a set-valued map by $\mathbf{h}=$ $(S, T, G r(\mathbf{h}))$, where $S$ is the source set, $T$ is the target set, and $G r(\mathbf{h})$ is the set $\{(s, t) \mid \exists s \in S$ with $\mathbf{h}(s)=t\}$. Now, given a set-valued map $\mathbf{h}$, we say that a sequence $\left\{\mathbf{h}_{j}\right\}_{j \in \mathbb{N}}$ of set valued maps with compact graph inward 
graph converges (denoted by $\mathbf{h}_{j} \stackrel{i g r}{\rightarrow} \mathbf{h}$ ) to $\mathbf{h}$ (also a set valued map with compact graph) if for every open subset $\Omega \subseteq S \times T$ such that $G r(\mathbf{h}) \subseteq \Omega$ there exists a $j_{\Omega} \in \mathbb{N}$ such that $\operatorname{Gr}\left(\mathbf{h}_{j}\right) \subseteq \Omega$ whenever $j \geq j_{\Omega}$. Moreover, we define the flow of $\mathbf{h}, \Phi^{\mathbf{h}}$, to be the set-valued map from $\Omega \times \mathbb{R}$ to $\Omega$ whose value, for a given $\left(x_{0}, t\right) \in \Omega \times \mathbb{R}$, is the set of all $y \in \Omega$ such that there exists an integral curve $x(t):[0, T] \rightarrow \Omega$ of $\mathbf{h}$ satisfying $x \in \mathbf{h}$ a.e. which is defined on some subinterval $[0, T] \subseteq \mathbb{R}$ such that $t \in[0, T]$, $x(0)=x_{0}$, and $x(t)=y$.

Definition 2.3. Let $X$ and $Y$ be metric spaces. A regular set-valued map from $X$ to $Y$ is a set valued map $\mathbf{f}$ such that for every compact subset $K \subset X$ the restriction $\mathbf{f} \mid K$ is a set valued map with compact graph, and is a limit, in the sense of inward graph convergence, of a sequence of continuous single-valued maps from $K$ to $Y$.

Definition 2.4. Let $\mathbf{f}: \mathbb{R}^{m} \longrightarrow \mathbb{R}^{n}$ be a set-valued map, and let $\Lambda$ be a nonempty compact subset of $\mathbb{R}^{n \times m}$. Let $S$ be a subset of $\mathbb{R}^{m}$. We define $\Lambda$ to be the generalized differential quotient (GDQ) of $\mathbf{f}$ at $(x, \mathrm{f}(x))$ in the direction of $S$ (denoted by $\Lambda \in G D Q(\mathbf{f}, x, \mathbf{f}(x), S)$ ), if for every positive real number $\delta$ there exist $U$ and $\mathbf{g}$ such that:

1. $U$ is a compact neighborhood of $x \in \mathbb{R}^{m}$ and $U \cap S$ is compact.

2. $\mathbf{g}$ is a regular set-valued map from $U \cap S$ to the $\delta$-neighborhood $\Lambda^{\delta}$ of $\Lambda$ in $\mathbb{R}^{n \times m}$.

3. $\mathbf{g}(x) \cdot x \subseteq \mathbf{f}(x)$ for every $x \in U \cap S$.

We should note that $G D Q$ theory is a generalized differentiation theory in the sense of [3]. Moreover, the $G D Q$ theory has a strong directional open mapping property which we use to prove controllability.

Now we will state and give the briefest possible sketch of a proof for our initial result, that $\mathbf{f}$ (as a set valued object) is a $G D Q$ of $\Phi^{\mathrm{f}}$.

Theorem 2.1. Assume $\Omega$ is an open subset of $\mathbb{R}^{n}, f$ is a MMDA vector field on $\Omega$ as in Definition 1.1, and $q_{*} \in \Omega$. Then the set $L^{\mathbf{f}}$, defined by

$$
L^{\mathbf{f}}\left(q_{*}\right)(v, r)=v+r f\left(q_{*}\right) \text { for } f \in \mathbf{f}, v \in \mathbb{R}^{n}, r \in \mathbb{R}(2.6)
$$

is a GDQ of the set-valued map $\Phi^{\mathbf{f}}$ at $\left(q_{*}, 0\right)$ in the direction of $\mathbb{R}^{n}$.

Sketch of Proof: We need only satisfy the properties of Definition 2.4. We can choose a $\delta$ neighborhood of $q_{*}$ to be $U$. We define the following:

$\Lambda\left(q-q_{*}, t\right)=\left\{L^{f}\left(q_{*}\right)\left(q-q_{*}, t\right)+E_{(q-q *, t), p-q-t f\left(q_{*}\right)}^{n+1, n}\left(q-q_{*}, t\right)\right\}$

such that $p \in \Phi^{f}, f \in \mathbf{f}$ and where $E_{x, y}^{m, n}(u)=\frac{\langle x, u>}{\|x\|^{2}} y$ for $x \in \mathbb{R}^{m}, y \in \mathbb{R}^{n}$, and $u \in \mathbb{R}^{m}$. We extend the definition of $\Lambda$ to $t=0$ by defining $\Lambda(q, t)=\left\{L^{f}\left(q_{*}\right)\right\}$ (that is, the set of $L^{f}$ with $f \in \mathrm{f}$ at $q_{*}$ ). The proof goes in three stages:

1. Show $\Lambda\left(q-q_{*}, t\right) \cdot\left(q-q_{*}\right) \subseteq \Phi^{\mathrm{f}}$ for a sufficiently small neighborhood of $q_{*} \in \mathbb{R}^{n}$.

2. Show $\Lambda$ is a map from neighborhoods of $q_{*}$ to $\Lambda^{\delta}$.

3. Show $\Lambda$ is regular.

1) Let $M \in \Lambda(q, t)$ and $(q, t)$ be in a sufficiently small $\epsilon$-ball of $\left(q_{*}, 0\right)$ (i.e. $(q, t) \in\left(q_{*}+B_{\epsilon_{1}}, B_{\epsilon_{2}}\right)$ with $B_{\epsilon}$ denoting a ball of $\epsilon$ radius and $\epsilon_{1}$ and $\epsilon_{2}$ sufficiently small). Then $q_{*}+M\left(q-q_{*}, t\right)=$

$$
\begin{gathered}
q_{*}+L^{f}\left(q_{*}\right)\left(q-q_{*}, t\right)+E_{\left(q-q_{*}, t\right), p-x-t f\left(q_{*}\right)}^{n+1, n}\left(q-q_{*}, t\right) \\
=q_{*}+q-q_{*}+t f\left(q_{*}\right)+p-q-t f\left(q_{*}\right)=p
\end{gathered}
$$

So $\Lambda\left(q_{*}, t\right) \cdot q \subseteq \Phi^{\mathbf{f}}$.

2) Now we show that $d\left(\Lambda, L^{\mathbf{f}}\left(q_{*}\right)\right)<\delta$, where $d(\cdot, \cdot)$ is the Haussdorff set distance function defined by:

$$
d(A, B)=\max \left(\max _{b \in B} \min _{a \in A} d(a, b), \max _{a \in A} \min _{b \in B} d(a, b)\right) .
$$

To do so, we again let $M \in \Lambda(q, t)$. Then,

$$
\sup \left\|M-L^{\mathbf{f}}\left(q_{*}\right)\right\|=\sup _{f \in \mathbf{f}} \frac{\left\|p-q-t f\left(q_{*}\right)\right\|}{\left(\left\|q-q_{*}\right\|^{2}+t^{2}\right)^{\frac{1}{2}}}
$$

This, in turn, we can show must be small for $t$ and $\left\|q-q_{*}\right\|$ sufficiently small, showing that $d\left(\Lambda, L^{\mathbf{f}}\left(q_{*}\right)\right)<\delta$. 3) We know that MMDA systems are almost everywhere differentiable. This implies that we can construct regularizations of $f$ at all points of discontinuity, where the regularization of the transition of $f$ from $f_{i}$ to $f_{j}$ at $q_{*}$ is defined by:

$$
f_{i j}^{\xi}=\int_{\mathbb{R}^{n}} \phi(h) f(x+\xi) d h
$$

where $\phi(h) \geq 0$, is $C^{\infty}, \int_{\mathbb{R}^{n}} \phi(h) d h=1, \phi(h)=0$ for $\|h\|>0$, and $\xi>0$. This forms a finite set of regularizations. We then use these regularizations to form the smooth single valued maps which must converge to $\Lambda$ as $\xi \rightarrow 0$.

What we now show is that $[f, g]$ from Definition 2.1 is a $G D Q$ of the map

$$
\Xi^{\mathbf{f}, \mathbf{g}}(q, \varepsilon)= \begin{cases}\psi^{f, g}(q, \sqrt{\varepsilon}, \sqrt{\varepsilon}) & \text { if } \varepsilon \geq 0 \\ \psi^{f, g}(q, \sqrt{-\varepsilon}, \sqrt{-\varepsilon}) & \text { if } \varepsilon \leq 0\end{cases}
$$

for $f \in \mathbf{f}$ and $g \in \mathbf{g}$, and where $\psi^{f, g}(x, t, s)=\Phi^{-s g} \circ \Phi^{-t f} \circ \Phi^{s g} \circ \Phi^{t f}(x)$ where $\circ$ is the composition operator.

Theorem 2.2. Assume $\Omega$ is an open subset of $\mathbb{R}^{n}, \mathrm{f}$ and $\mathrm{g}$ are $M M D A$ vector fields on $\Omega$ as in Definition 1.1, and $q_{*} \in \Omega$. Then the set $\left\{L^{w}\left(q_{*}\right) \mid w \in[\mathbf{f}, \mathbf{g}]\right\}$ is a $G D Q$ of the map $\Xi^{\mathbf{f}, \mathbf{g}}$ at $\left(q_{*}, 0\right)$ in the direction of $\mathbb{R}^{n}$.

Very Brief Sketch of Proof: The proof of this statement is significantly more involved than the proof of 
Theorem 2.1, and we therefore only give it in the broadest details. As in the previous proof, the only case of interest to us is $q_{*}$ at a point of discontinuity of $\sigma$. At such a point we know that $\dot{x} \in \mathbf{f} u_{1}+\mathbf{g} u_{2}$. where $\mathbf{f}=\operatorname{co}\left\{f_{i}\right\}$ and $\mathbf{g}=\operatorname{co}\left\{g_{j}\right\}$. As in the previous proof we choose regularizations of $f$ and $g, f_{i j}$ and $g_{k l}$, which form, for every value of $\xi$, a finite set of $C^{\infty}$ maps with which one can take classical lie brackets. We then show that this set of lie brackets inward graph converge to $[f, g]$ as defined in Definition 2.1. In particular, we show that

$$
\begin{array}{r}
\sup _{M \in L^{u^{*}}\left(q_{*}\right)}\left\|\Xi^{f, g}(q, \varepsilon)-\Xi^{f, g}\left(q_{*}, 0\right)-M\left(q-q_{*}, \varepsilon\right)\right\| \\
=\mathcal{O}\left(\varepsilon+\left\|q-q_{*}\right\|\right)
\end{array}
$$

This then satisfies the sufficient condition given in [18] for a set to be a GDQ of a map.

Having shown both of those are GDQs of their respective maps, we can use the considerable machinery developed in [17] to prove controllability using an open mapping theorem developed therein. This leads us to the following sufficient condition for MMDA systems to be controllable.

Theorem 2.3. Let $\Sigma$ be a control system as in Definition 1.1 , and $q_{*}$ be a point such that $\bar{\Delta}\left(q_{*}\right)=\mathbb{R}^{n}$. Then $\Sigma$ is small time locally controllable at $q_{*}$

The proof is nearly the same as that found in [16]. It relies on constructing a map composing flows of all the MMDA fields and their associated brackets. Then one uses the theory in [17] to show that this map is open, thus showing controllability.

\section{The Example}

Here we consider the relatively simple example of a six wheeled vehicle such as that found in Fig. 1 and apply the result of Section 2. This overconstrained wheeled vehicle is a simplified model of the six-wheeled rockerbogey mobility system of the Sojourner vehicle that landed on Mars in 1997. This chassis geometry will also be the basis for near-term Mars rover missions.

This system is "overconstrained" because not all the non-holonomic kinematic wheel constraints can be simultaneously satisfied. Consequently, the vehicle's motion can not be determined directly from kinematic constraints (i.e., it's governing equations of motion can not be put in the form $\dot{q}=\sum_{i} g_{i}(q) u_{i}$ with the $g_{i}$ smooth). Except when the vehicle moves straight ahead, at least one of the non-powered wheels must be slipping at all times. Hence, classical nonholonomic control theories do not apply to this vehicle. In [12] we proposed a power dissipation method for determining the governing equations of such overconstrained systems when they are moving slowly.
Definition 3.1. The Dissipation or Friction Functional for an $n$-contact state is defined to be

$$
\mathcal{D}=\sum_{i=1}^{n} \alpha_{i}\left|\omega^{i}(q) \dot{q}\right|
$$

where $q$ represents the configuration, $\alpha_{i}=\mu_{i} F_{i}$, with $\mu_{i}$ and $F_{i}$ being the Coulomb friction coefficient and normal force at the $i^{\text {th }}$ wheel/ground contact, which are assumed known. $\omega^{i}(q) \dot{q}$ represents the velocity of the $i^{\text {th }}$ wheel's point of contact with the ground.

Since one or more of the contact points must always be in a slipping state due to the overconstrained geometry, the power dissipation approach states that the vehicle's motion at any instant is the one that minimizes $\mathcal{D}$, the power lost to slip. In [12] we showed that the minimum of power dissipation function yields governing equations that are MMDA systems (Definition 1.1). The power dissipation function measures the object's total energy dissipation due to contact slippage. It essentially restricts attention to those Lagrangian states which can be reduced to kinematic systems. See [8] for some details of such a reduction in the smooth case.

Section 3.1 discusses the full input space for a sixwheeled, fully actuated system. The complexity of such a system leads us to do an input state reduction to two inputs which we discuss in Section 3.2. In Section 3.3, as an example of how poor choices can be made in such a reduction, we attempt to gain more actuation by allowing both front wheels to be independently steerable and then show that such a system does not satisfy the sufficient conditions for controllability.

\subsection{Six wheels and two steerable wheels}

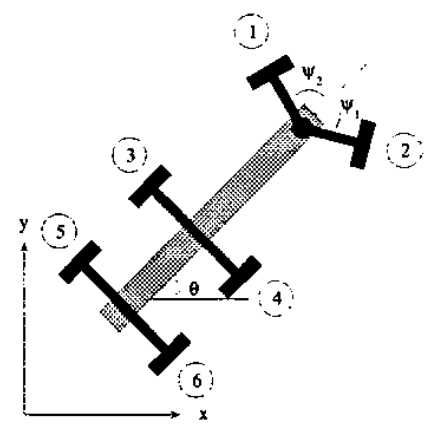

Figure 1: 6 driven wheels, 2 steerable wheels

First we consider a full kinematic model of the Rocky 7 Sojourner robot. It has two steerable front wheels (inputs $u_{1}, u_{2}$ ) and all 6 of its wheels are driven (inputs $\left.u_{3}, \ldots, u_{8}\right)$. We will assume it is on flat ground with only the coefficient of friction altering the contact state. We idealize the steering of the wheels as a rotation about a vertical axis. Additionally, let the distance from that axis to the middle axle be $l$, and the distance from the middle axle to the back axle be $r$. In 
this model, we naively have a total of 12 nonholonomic constraints on the system, with each wheel contributing a "no side way slip" constraint and a "no rolling" constraint. Clearly, not all of these constraints can be simultaneously satisfied except in non-generic cases, 1) the two rear axles are parallel, and therefore can only accommodate forward motion without slipping, and 2) there is no a priori reason to believe that the inputs $u_{i}$ will produce mutually compatible velocities. Thus, the system is overconstrained. Applying the PDM to this system, we get

$$
\left(\begin{array}{c}
12 \\
3
\end{array}\right)=\frac{12 !}{9 ! 3 !}=220
$$

kinematic states. That is, there are 220 different combinations of slipping motions. This is clearly unacceptable both from a complexity and practical standpoint.

To make progress on the analysis front, we reduce the number of inputs by introducing "matching" constraints. Observe that for any choice of $u_{1}$ and $u_{3}$ one can choose the other $u_{i}$ inputs to be kinematically compatible with the motion produced by $u_{1}$ and $u_{3}$. Therefore, we reduce the dimension of the input space by requiring the following to hold:

$$
\begin{aligned}
& u_{6}=A d_{g_{63}}^{[2]} u_{3} \\
& u_{7}=A d_{g_{73}}^{[2]} u_{3} \\
& u_{8}=A d_{g_{3}}^{[2]} u_{3} \\
& u_{5}=A d_{g_{53}}^{[2]} u_{3} \\
& u_{4}=A d_{g_{43}}^{[2]} u_{3} \\
& u_{2}=A d_{g_{12}}^{[2]} u_{1}
\end{aligned}
$$

where $A d_{g_{i j}}^{k j}$ is the $k^{t h}$ component of the Adjoint.operator of the rigid body transformation going from frame $i$ (associated with the point where the input $u_{i}$ acts on the system) to frame $j$. Practically speaking, these constraints enforce the two front wheels to steer tcgether, and the remaining wheels to have a compatible motion. Therefore, $u_{1}$ and $u_{3}$ completely determine all the other control inputs. One can think of this system as being driven by two "virtual" inputs, $v_{1}$ and $v_{2}$ so as to get an underactuated vehicle. This effectively determines the model in Section 3.2. This technique is essentially an ad-hoc reduction in the shape space instead of the traditional group space reduction.

\subsection{Reduction 1}

Here we take the reduction as posed in the previous section. In this example, the front wheel is driven and steerable, while the middle and back wheels are passive. Additionally, the front wheel is always assumed to be in contact with the ground.

Using the power dissipation approach, one can show that the minimum of $\mathcal{D}$ must occur when either the middle or back wheel slips. If the vehicle configuration is

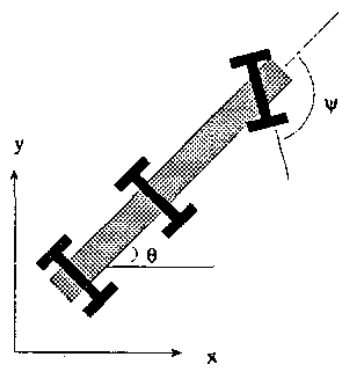

Figure 2: 1 driven and steered wheel, 2 passive wheels $q=[x, y, \theta]^{T}$ and the controls $u_{1}$ and $u_{2}$ are associated with the drive and steering velocities respectively, the vehicle's governing equations of motion are:

$$
\begin{aligned}
& \dot{q}=g_{\sigma_{1}}(q) u_{1}+g_{3}(q) u_{2} \quad \sigma_{1}:(q, t) \rightarrow\{a, b\} \\
& g_{1 a}=\left[\begin{array}{llll}
\cos (\psi) \cos (\theta) & \cos (\psi) \sin (\theta) & \frac{1}{l} \sin (\psi) & 0
\end{array}\right]^{T} \\
& g_{1 b}=\left[\begin{array}{c}
\cos (\theta) \cos (\psi)-\frac{r \sin (\theta) \sin (\psi)}{l+r} \\
\cos (\psi) \sin (\theta)+\frac{r \cos (\theta) \sin (\psi)}{l+r} \\
\frac{1}{l+r} \sin (\psi) \\
0
\end{array}\right] \\
& g_{3}=\left[\begin{array}{llll}
0 & 0 & 0 & 1
\end{array}\right]^{T}
\end{aligned}
$$

The function which determines the switching boundaries is:

$$
\Psi(q)=\left(\frac{F_{1} \mu_{1}}{F_{2} \mu_{2}}\right)^{2}\left(\frac{l-r}{r}\right)^{2}-1 .
$$

where $F_{i}$ are the normal forces above the middle axis and back axis, and the $\mu_{i}$ are the coefficients of friction at the two rear wheel contacts. When $\Psi(q)>0, \sigma_{1}=a$; when $\Psi(q)<0, \sigma_{1}=b$. Therefore switching is determined by $q \in Q$ such that $\Psi(q)=0$. Intuitively, variations in tire-ground friction and vehicle weight distribution can cause alternations in the choice of the slipping wheel.

Controllability is determined by the rank of the distribution:

$$
\left(g_{3}, g_{\sigma_{1}},\left[g_{3}, g_{\sigma_{1}}\right],\left[\left[g_{3}, g_{\sigma_{1}}\right], g_{\sigma_{1}}\right]\right)
$$

Computing accordingly, we get $\left[g_{\sigma_{1}}, g_{3}\right]=$

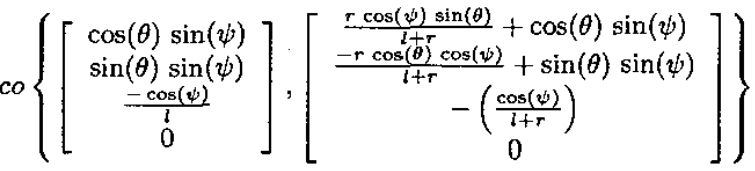

$$
\begin{aligned}
& {\left[g_{\sigma_{1}},\left[g_{\sigma_{1}}, g_{3}\right]\right]=\operatorname{and}\left\{\left[\begin{array}{c}
\frac{-1}{l} \sin (\theta) \\
\frac{1}{l} \cos (\theta) \\
0 \\
0
\end{array}\right],\left[\begin{array}{c}
\frac{-1}{l+r} \sin (\theta) \\
\frac{1}{l+r} \cos (\theta) \\
0 \\
0
\end{array}\right]\right\}}
\end{aligned}
$$

Now, substituting in the algebraic equivalent for $\operatorname{co}\{\cdot, \cdot\}$ we can evaluate the determinant of Equation 3.2:

$\left[g_{3}, \operatorname{co}\left\{g_{1 a}, g_{1 b}\right\},\left[g_{3}, \operatorname{co}\left\{g_{1 a}, g_{1 b}\right\}\right],\left[\left[g_{3}, c o\left\{g_{1 a}, g_{1 b}\right\}\right], c o\left\{g_{1 a}, g_{1 b}\right\}\right]\right]$ $=\left[g_{3}, c o\left\{g_{1 a}, g_{1 b}\right\}, c o\left\{\left[g_{3}, g_{1 a}\right],\left[g_{3}, g_{1 b}\right]\right\}\right.$, $\left.\operatorname{co}\left\{\left[g_{1 a},\left\{g_{1 a}, g_{3}\right]\right],\left[g_{1 b},\left[g_{1 b}, g_{3}\right]\right]\right\}\right]$

$=\left[g_{3}, \delta_{1} g_{1 a}+\left(1-\delta_{1}\right) g_{1 b}, \delta_{2}\left[g_{3}, g_{1 a}\right]+\left(1-\delta_{2}\right)\left[g_{3}, g_{1 b}\right]\right.$, $\left.\delta_{3}\left[g_{1 a},\left[g_{1 a}, g_{3}\right]\right]+\left(1-\delta_{3}\right)\left[g_{1 b},\left[g_{1 b}, g_{3}\right\}\right]\right]$ 
where $\delta_{i} \in[0,1]$ for $i=1,2,3$. The determinant is

$=\frac{\left(l+r \delta_{3}\right)\left(2 l+r\left(\delta_{1}+\delta_{2}\right)+r\left(\delta_{2}-\delta_{1}\right) \cos (2 \psi)\right)}{2 l^{2}(l+r)^{2}}$

which equals 0 only if $\delta_{3}<0$ which is not an admissible $\delta_{3}$. Hence, the vehicle is always STLC, as expected. Physically, this result implies that the vehicle remains locally controllable even as the status of slipping wheel alters unexpectedly.

\subsection{Reduction 2}

It is clearly necessary that all of the individual models in an MMDA system be controllable in order for the condition in Theorem 2.3 to be satisfied. Now we ask, is it sufficient that all of the individual models in an MMIDA system be controllable? If it is, then we need only concern ourselves with the analysis of the individual smooth models. The answer to this question, perhaps surprisingly, is no. This example illustrates why.

We now consider the case where the front two wheels are independently steered. This choice, though seemingly reasonable, will cause the resulting system to fail our controllability test. To illustrate this concept, we consider a vehicle with the same two front steerable wheels as the Rocky 7 , but with only one back wheel for simplicity (Fig. 3). For simplicity we assume that

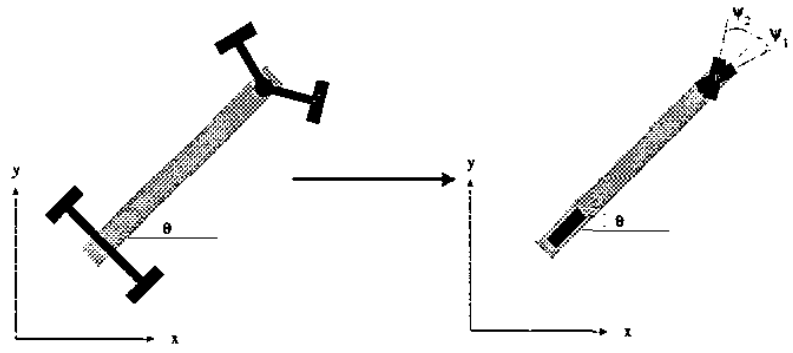

Figure 3: 2 driven and steered wheels, 1 passive wheel

the two front wheels are collapsed down along their respective axes so that they both are connected at the same point (see Figure 3). (This simplification does not change the outcome of the analysis significantly.) We will see that this vehicle is only controllable when $\psi_{1}=\psi_{2}$ (when it is indistinguishable from a kinematic car). An analysis of this system using the PDM shows that there are two distinct kinematic states for this vehicle (i.e., two different permutations of wheel slip). The governing equations of both slipping cases look like kinematic cars, i.e.,

$$
\dot{q}=g_{\sigma_{1}}(q) u_{1}+g_{3}(q) u_{2} \quad \sigma_{1}:(q, t) \rightarrow\{a, b\}
$$

where

$g_{1 a}=\left[\begin{array}{c}\cos \psi_{1} \cos \theta \\ \cos \psi_{1} \sin \theta \\ \sin \psi_{1} \\ 0\end{array}\right], g_{1 b}=\left[\begin{array}{c}\cos \psi_{2} \cos \theta \\ \cos \psi_{2} \sin \theta \\ \sin \psi_{2} \\ 0\end{array}\right], g_{3}=\left[\begin{array}{l}0 \\ 0 \\ 0 \\ 1\end{array}\right]$

As before in Equation (3.2), controllability is determined by the rank of:

$$
\left(g_{3}, g_{\sigma_{1}},\left\lfloor g_{3}, g_{\sigma_{1}}\right],\left[\left[g_{3}, g_{\sigma_{1}}\right], g_{\sigma_{1}}\right]\right)
$$

Computing, we get:

$$
\left[g_{3}, g_{\sigma_{1}}\right]=c o\left\{\left[\begin{array}{c}
\cos \psi_{1} \sin \theta \\
\cos \psi_{1} \cos \theta \\
-\cos \psi_{1} \\
0
\end{array}\right],\left[\begin{array}{c}
\cos \psi_{2} \sin \theta \\
\cos \psi_{2} \cos \theta \\
-\cos \psi_{2} \\
0
\end{array}\right]\right\}
$$

and

$$
\left[\left[g_{3}, g_{\sigma_{1}}\right], g_{\sigma_{1}}\right]=\left[\begin{array}{c}
-\sin \theta \\
\cos \theta \\
0 \\
0
\end{array}\right]
$$

Evaluating the determinant as we did before, we now compute if there exists a selection of the differential inclusion such that this is not full rank, i.e., we need to find out if there exists $\delta_{1}, \delta_{2}$ such that

$$
\begin{array}{r}
\operatorname{det}\left[g_{3}, \delta_{1} g_{1 a}+\left(1-\delta_{1}\right) g_{1 b}, \delta_{2}\left[g_{3}, g_{1 a}\right]+\left(1-\delta_{2}\right)\left[g_{3}, g_{1 b}\right]\right. \\
\left.\delta_{3}\left[g_{1 a},\left[g_{1 a}, g_{3}\right]\right]+\left(1-\delta_{3}\right)\left[g_{1 b},\left[g_{1 b}, g_{3}\right]\right]\right]=0
\end{array}
$$

Computing, we find that the above determinant equals

$$
\delta_{1}+\delta_{2}-2 \delta_{1} \delta_{2}-1+\left(\delta_{1}+\delta_{2}-2 \delta_{1} \delta_{2}\right) \cos \left(\psi_{1}-\psi_{2}\right)
$$

Now, if

$$
\chi\left(\delta_{1}, \delta_{2}\right) \stackrel{\text { def }}{=} \frac{1}{\delta_{1}+\delta_{2}-2 \delta_{1} \delta_{2}}-1
$$

then the controllability condition depends on whether there exists $\delta_{i}$ such that $\chi\left(\delta_{1}, \delta_{2}\right) \in[-1,1]$. Clearly there do exist such $\delta_{i}$, just choose $\delta_{1}=0$ and $\delta_{2}=1$. Then $\chi=0$. However, one may ask if making $\left|\psi_{1}-\psi_{2}\right|$ sufficiently small helps. Doing so changes the requirement to $\chi \in[-\varepsilon, \varepsilon]$, which again has a solution for the same choice of $\delta_{i}$. Therefore, this vehicle design is arbitrarily sensitive to switching with respect to local controllability. That is, although on the one hand it is desirable to get extra actuation by controlling $\psi_{1}$ and $\psi_{2}$ independently, on the other hand it is desirable to have a model we can show is controllable.

Now, what if the selections of $\left[\dot{g}_{\sigma_{1}}, g_{3}\right]$ and $\left[\left[g_{\sigma_{1}}, g_{3}\right], g_{3}\right]$ are coupled in some way? Consider the situation where, if we again parameterize the selections of $\left[g_{\sigma_{1}}, g_{3}\right]$ by $\delta_{1} \in[0,1]$ and the selections of $\left[\left[g_{\sigma_{1}}, g_{3}\right], g_{3}\right]$ by $\delta_{2} \in[0,1]$, what if $\delta_{2}=h\left(\delta_{1}\right)$. For example, take $h$ to be the identity. Then

$$
\chi\left(\delta_{1}, \delta_{2}\right)=\frac{1}{2 \delta_{1}-2 \delta_{1}^{2}}-1
$$


which only has a solution in $[-1,1]$ for $\delta_{1}=\frac{1}{2}$. Therefore, if we allow a slightly weaker notion of controllability by only requiring that the system be controllable almost always, this system would then be controllable. The key point here is that this system does not satisfy the requirements for controllability. This has two implications. First, it is a weakness in the theory, because we also do not know that the system is not locally controllable. Second, it indicates that we should avoid algorithm designs that rely on a paramterization like that found in Figure 3 until we have developed analytical tools that make such a system tractable.

\section{Conclusions}

This paper extends recent controllability results for MMDA systems that can be viewed as extensions of Chow's classical controllability theorem. This paper additionally studied an important MMDA example where the individual plants are driftless affine. Moreover, the variations of the model studied in Section 3.3 illustrate that controllability of the individual models that make up an MMDA system is not sufficient to guarantee controllability of the overall MIMDA system. Because of the potential importance of the vehicles discussed in Section 3 in future planetary exploration missions, future work will investigate algorithms for stabilizing the multimodel systems of Definition 1.1. In particular, we will use the formalism of $G D Q$ s to study stability. That said, showing stability of such systems is complicated by a number of factors. Stability is difficult even for underactuated mechanical system with smooth dynamics. Stability theorems have often used significant insights into geometry (see $[7,9,19]$ ). For systems like those discussed in this paper, such geometric techniques must be extended to nonsmooth systems, which will inevitably be a formidable task.

Acknowledgements: This work was partially supported by the National Science Foundation (grant NSF9402726) through its Engineering Research Center (ERC) program.

\section{References}

(1) E. Asarin, O. Bournez, T. Dang, O. Maler, and A. Pneuli. Effective synthesis of switching controllers for linear systems. Proc. IEEE, 88(7):1011-1025, July 2000 .

[2] M.S. Branicky. Multiple Lyapunov functions and other analysis tools for switched and hybrid systems. IEEE Trans. Automatic Control, 43(4):475-482, April 1998.

[3] F.H. Clarke, Y.S. Ledyaev, R.J. Stern, and P.R. Wolenski. Nonsmooth Analysis and Control Theory. Springer, 1998.
[4] W.P. Dayawansa and C.F. Martin. A converse Lyapunov theorem for a class of dynamical systems which undergo switching. IEEE Trans. Automatic Control, 44 (4):751-760, Apr. 1999.

[5] A.F. Filippov. Differential Equations with Discontinuous Right-Hand Sides. Kluwer, 1988.

[6] J.P. Hespanha, D. Liberzon, and A.S: Morse. Logicbased switching control of a nonholonomic system with parametric uncertainty. Systems Control Lett., 38:167$177,1999$.

[7] I. Kolmanovsky and N.H. McClamroch. Developments in nonholonomic control problems. IEEE Control Systems Magazine, pages 20-36, December 1995.

[8] A.D. Lewis. When is a mechanical control system kinematic? In Proc. $38^{\text {th }}$ IEEE Conf. on Decision and Control, pages 1162-1167, Dec. 1999.

(9) A.D. Lewis, R.M. Murray, and J.W. Burdick. Nonholonomic mechanics and locomotion: the snakeboard example. In Proc. IEEE Int. Conf. on Robotics and Automation, pages 2391-2397, May 1994.

[10] D. Liberzon and A.S. Morse. Basic problems in stability and design of switched systems. IEEE Control System Mag., 19(5):59-70, 1999.

[11] K. A. Morgansen, V. Duindam, R. J. Mason, J. W. Burdick, and R. M. Murray. Nonlinear control methods for planar carangiform robot fish locomotion. In Proc. IEEE Int. Conf. on Robotics and Automation, Seoul, Korea, 2001.

[12] T. D. Murphey and J. W. Burdick. Issues in controllability and motion planning for overconstrained wheeled vehicles. In Proc. Int. Conf. Math. Theory of Networks and Systems (MTNS), Perpignan, France, 2000.

[13] T. D. Murphey and J. W. Burdick. A controllability test and motion planning primitives for overconstrained vehicles. In Proc. IEEE Int. Conf. on Robotics and Au. tomation, Seoul, Korea, 2001.

[14] T. D. Murphey and J. W. Burdick. A controllability test for nonlinear multiple model systems. In Proc. IEEE American Controls Conference ( $A C C$ ), Anchorage, Alaska, 2002.

[15] G.J. Pappas, G. Laffierier, and S. Sastry. Hierarchically consistent control sytems. IEEE Trans. Automatic Control, 45(6):1144-1160, June 2000.

[16] F. Rampazzo and H. J. Sussmann. Set-valued differentials and a nonsmooth version of Chow's theorem. In Proc. of 40 th Conf. Decision Control, 2001.

[17] H.J. Sussmann. Multidifferential calculus: Chain rule, open mapping and transversal intersection theorems. In Optimal Control: Theory, Algorithms, and Applications, pages 436-487. Kluwer, 1998.

[18] H.J. Sussmann. New theories of set-valued differentials and new versions of the maximum principle of optimal control theory, chapter Nonlinear Control in the Year 2000. Springer-Verlag, London, 2000. A. Isidori, F. LamnabhiLagarrigue, and W. Respondek, Eds.

[19] A.R. Teel, R.M. Murray, and G. Walsh. Nonholonomic control systems: From steering to stabilization with sinusoids. Int. $J$. of Control, 62(4):849-870, 1995.

[20] M. Zefran and J.W. Burdick. Design of switching controllers for systems with changing dynamics. Proc. Conf. on Decision and Control, 1998. 